\title{
Interference Mitigation for the GPS Receiver Utilizing the Cyclic Spectral Analysis and RR-MSWF Algorithm
}

\author{
Yi HU ${ }^{1}$, Maozhong SONG ${ }^{2}$, Xiaoyu $D A N G^{2}$, Hongli YAN ${ }^{1}$ \\ ${ }^{1}$ School of Electronics \& Electrical Engineering, Chuzhou University, Chuzhou, China \\ ${ }^{2}$ School of Electronics \& Information Engineering, Nanjing University of Aeronautics \& Astronautics, Nanjing, China
}

\{hygps607,yhli81\}@163.com, \{smz106,dang\}@nuaa.edu.cn

Submitted October 1, 2016 / Accepted April 24, 2017

\begin{abstract}
A method utilizing the cyclic spectral analysis (CSA) and reduced-rank multistage Wiener filtering (RR$M S W F)$ algorithm to mitigate the interference for the GPS receiver is proposed. In many cases, interference from adjacent channel or from cochannel overlaps on the weak global positioning system (GPS) signal in both time and frequency domains, and it is hard to mitigate this kind of strong interference with the conventional filtering techniques. While with the proposed method given in the paper, we can mitigate the interference effectively. The general process of the proposed method is that first we get the cyclic frequencies (CFs) of the strong interference by CSA of the received GPS signal. And then with the obtained CFs of the interference, we use the blind adaptive frequency shift (BA-FRESH) filter to get the principal process of mitigating the strong interference and separating the weak GPS signal. Finally by utilizing the efficient RR-MSWF algorithm to implement the BA-FRESH filtering, we can mitigate the strong interference effectively and hence improve the performance of the GPS receiver.
\end{abstract}

\section{Keywords}

GPS, interference mitigation, cyclic spectral analysis, frequency shift filtering (FRESH), reduced-rank multistage Wiener filtering algorithm (RR-MSWF)

\section{Introduction}

The development of modern wireless communication systems and the emergence of many personal privacy devices (PPDs) make the working environment of the GPS receiver more severe and more complicated than ever [1-3]. The adjacent channel interference and/or the cochannel interference from these systems and/or from PPDs may cause the GPS receiver serious performance degradation when their strength is much stronger than that of the GPS signal. For the adjacent channel interference, it mainly arises from the communication satellite or from the wireless mobile communication system whose transmitting frequency or harmonics is close to the frequency of GPS signal [4-6]. While for the cochannel interference, it mainly arises from the other satellite navigation system such as Galileo or quasi-zenith satellite system (QZSS) which shares the same downlink frequency with the GPS signal [1]. For example, both GPS L1 C/A code signal and Galileo E1 OS signal have the downlink frequency of 1575.42 MHz. In addition, except these two types of unintentional interference, the GPS receiver may also be subject to the intentional interference from the hostile such as multi-tone interference [2], narrowband continuous wave interference [7], [8], linear frequency modulation interference [9] and so on. Though the nature of direct-sequence spread-spectrum (DSSS) of the GPS signal can reject the interference in some extent, when the strength of the interference becomes relatively stronger, the acquisition and tracking performance of the GPS receiver will degrade greatly, and this will correspondingly degrade the accuracy of positioning and ranging of the GPS receiver [10].

To mitigate the interference mentioned above, many filtering techniques such as matched filtering (MF) [11], finite impulse response (FIR) filtering [12], FFT-based interference excision method [13] and so on have been proposed. Though by these filtering techniques we can mitigate the interference overlapping on the GPS signal in time or frequency domain, in many cases the interference may overlap on the weak GPS signal in both time and frequency domains, and we cannot effectively mitigate them any more by these conventional filtering techniques. To solve this problem, methods such as short time Fourier transform (STFT) [4], wavelet transform (WT) and related methods [14] are proposed. For STFT, WT and related methods, their performance of interference mitigation often depends on the selection of transforming windows or wavelet basis functions, and this may bring some inconvenience in practical applications. On the other hand, for most man-made signals including the GPS signal and interference that we are to mitigate, they are spectral correlated in their corresponding cyclic spectral domains [15-17], and this feature can be used to mitigate the strong interference. Based on this, a new method utilizing CSA [18-20] of the received GPS signal and RR-MSWF algorithm [21], [22] to mitigate the strong interference for the GPS receiver is proposed. The general process of the proposed method is 
that first we get the CFs of strong interference by CSA of the received GPS signal. And then with the obtained CFs, we use the BA-FRESH filter [23-25] to get the principal process of mitigating the strong interference and separating the weak GPS signal. Finally by utilizing the efficient RRMSWF algorithm to implement the BA-FRESH filtering, we can effectively mitigate the strong interference and hence improve the performance of the received GPS signal.

The rest of the paper is organized as follows. In Sec. 2, CSA of the received GPS signal overlapped with strong interference is given. Section 3 presents the detailed interference mitigation process with BA-FRESH filter and RR-MSWF algorithm based on the results given in Sec. 2. In Sec. 4, simulations on CSA of the received GPS signal, acquisition and tracking performance of the separated GPS signal are offered. Finally, the paper is concluded in Sec. 5.

\section{CSA of the Received GPS Signal}

\subsection{Signal Model}

Considering that single coarse acquisition $(\mathrm{C} / \mathrm{A})$ code signal is acquired and tracked in one channel of the GPS receiver, the received GPS L1 C/A code signal overlapped with interference after down-conversion can be given as [3]

$$
x(t)=A_{\mathrm{s}} s(t) \cos \left[2 \pi\left(f_{\mathrm{IF}}+f_{\mathrm{d}}\right) t+\phi_{\mathrm{s}}\right]+I(t)+n(t)
$$

where $A_{\mathrm{S}}$ and $\Phi_{\mathrm{S}}$ are the amplitude and initial phase of the carrier, respectively; $f_{\mathrm{IF}}$ is the intermediate frequency (IF) of the GPS receiver and $f_{\mathrm{d}}$ is the Doppler shift; $n(t)$ is assumed the additive white Gaussian noise; $I(t)$ is the interference, $s(t)$ is the baseband signal of GPS and

$$
s(t)=\sum_{n=-\infty}^{+\infty} b_{\mathrm{g}}(n) \sum_{m=0}^{M-1} c_{m} p_{T_{c}}\left(t-\tau_{\mathrm{g}}-m T_{\mathrm{c}}-n T_{\mathrm{g}}\right)
$$

where $b_{\mathrm{g}}(n)$ is the data bit with value \pm 1 with equal probability; $\left\{c_{m}\right\}_{m=0}^{M-1}$ is one cycle of $\mathrm{C} / \mathrm{A}$ code spreading sequence with length $M$, and $c_{m} \in\{ \pm 1\} ; \tau_{\mathrm{g}}$ is the initial phase of GPS C/A code; $T_{\mathrm{g}}$ and $T_{\mathrm{c}}$ are the cycles of the data bit and $\mathrm{C} / \mathrm{A}$ code chip, respectively; $p_{T_{c}}(\bullet)$ is a unit-height rectangular pulse whose support is on $\left[-T_{\mathrm{c}} / 2, T_{\mathrm{c}} / 2\right]$.

For convenience, we make two hypotheses on $I(t)$ of (1): (i) there has one strong interference in $I(t)$. In fact, if there has more than one strong interference, we can repeat the interference mitigation process with the proposed method till all the strong interference is cancelled; (ii) due that the paper is mainly focused on the interference mitigation by CSA method, the recognition of interference is out the scope of this paper (cf. [26], [27] if needed), and here we assume that the type of interference we are to mitigate is known. Based on these two hypotheses and without loss of generality, we choose a binary phase shift keying (BPSK) signal as $I(t)$ due that it has both the CFs related to the carrier frequency and the CFs related to the bit/baud rate [22] which are the two key elements for the following BA-FRESH filter, thus $I(t)$ can be written as

$$
\begin{aligned}
I(t) & =A_{i} i(t) \cos \left[2 \pi\left(f_{\mathrm{IF}}+\Delta f_{i}\right) t+\phi_{i}\right] \\
& =A_{i}\left(\sum_{n=-\infty}^{+\infty} b_{i}(n) p_{T_{i}}\left(t-n T_{i}\right)\right) \cos \left[2 \pi\left(f_{\mathrm{IF}}+\Delta f_{i}\right) t+\phi_{i}\right]
\end{aligned}
$$

where $i(t)$ is the baseband signal of $I(t) ; \Delta f_{i}$ is the frequency bias relative to $f_{\mathrm{IF}} ; b_{i}(n), T_{i}, \phi_{i}$ and $p_{T_{i}}(\bullet)$ have the similar meanings as those given in (1) and (2).

\subsection{CSA of the Received GPS Signal}

For the received GPS signal $x(t)$ given in (1), its cyclic autocorrelation function (CAF) and the corresponding spectral correlation function (SCF) can be given as [23]

$$
\begin{gathered}
R_{x}^{\alpha}(\tau)=\left\langle x(t+\tau / 2) x^{*}(t-\tau / 2) \exp (-\mathrm{j} 2 \pi \alpha t)\right\rangle, \\
S_{x}^{\alpha}(f)=\lim _{T \rightarrow \infty} T\left\langle X_{T}(t, f+\alpha / 2) X_{T}^{*}(t, f-\alpha / 2)\right\rangle
\end{gathered}
$$

where the superscript "**" denotes complex conjugate, $\tau$ is the time lag, $\alpha$ is the $\mathrm{CF}$ of $x(t)$ and $\alpha \neq 0,\langle\bullet\rangle$ denotes the time average on $t$, and

$$
X_{T}(t, v)=\frac{1}{T} \int_{t-T / 2}^{t+T / 2} x(u) \exp (-\mathrm{j} 2 \pi v u) \mathrm{d} u .
$$

For $R_{x}^{\alpha}(\tau)$ and $S_{x}^{\alpha}(f)$, they are actually a Fourier transform pair, i.e.,

$$
S_{x}^{\alpha}(f)=\int_{-\infty}^{\infty} R_{x}^{\alpha}(\tau) \exp (-\mathrm{j} 2 \pi f \tau) \mathrm{d} \tau .
$$

For the conjugate CAF of $x(t)$ and its SCF, they have the similar relationships as (4) to (7). In addition, the discrete CAF and SCF corresponding to $x(t)=x\left(n T_{\mathrm{S}}\right)$ are respectively as [15]

$$
\begin{gathered}
R_{x}^{\alpha}(k)=\lim _{N \rightarrow \infty} \frac{1}{2 N+1} \sum_{n=-N}^{N} R_{x}((n+k), n) \exp \left(-\mathrm{j} 2 \pi \alpha(n+k / 2) T_{\mathrm{S}}\right) \\
S_{x}^{\alpha}(f)=\sum_{k=-\infty}^{+\infty} R_{x}^{\alpha}(k) \exp \left(-\mathrm{j} 2 \pi k T_{\mathrm{s}} f\right)
\end{gathered}
$$

where $R_{x}(n, m)$ is the conventional autocorrelation function of $x(n)$, and $T_{\mathrm{S}}=1 / f_{\mathrm{S}}$ is the sampling time interval.

Substituting (1) and (3) into (4) to (7) or (8) to (9), we can get the CFs of $x(t)$ from $S_{x}^{\alpha}(f)$. But often the strength of interference is stronger than that of the GPS signal, the cyclic spectrum of GPS signal may be concealed by that of the interference, thus we can get the CFs of interference first, and then cancel the interference from $x(t)$ with the following BA-FRESH filter using the obtained CFs. Based on (1) the SCF of $I(t)$ can be given as [23] 


$$
S_{I}^{\alpha}(f)=\left\{\begin{array}{l}
\frac{A_{i}^{2}}{4 T_{i}}\left[\begin{array}{l}
\left.Q\left(f+f_{i}+\frac{\alpha}{2}\right) Q^{*}\left(f+f_{i}-\frac{\alpha}{2}\right)+\right] \\
Q\left(f-f_{i}+\frac{\alpha}{2}\right) Q^{*}\left(f-f_{i}-\frac{\alpha}{2}\right)
\end{array}\right], \alpha=\frac{k}{T_{i}} \\
\frac{A_{i}^{2}}{4 T_{i}} Q\left(f \pm f_{i}+\frac{\alpha}{2}\right) Q^{*}\left(f \mp f_{i}-\frac{\alpha}{2}\right) \mathrm{e}^{ \pm \mathrm{j} 2 \phi_{i}}, \alpha=\mp 2 f_{i}+\frac{k}{T_{i}} \\
0, \quad \text { otherwise }
\end{array}\right.
$$

where $\alpha$ is the CF of $I(t), k \in \mathbb{Z}, f_{i} \triangleq f_{\mathrm{IF}}+\Delta f_{i}$ and $Q(f)=\sin \left(\pi f T_{i}\right) /(\pi f)$.

With (10) we can get the amplitude of $S_{I}^{\alpha}(f)$ at $f=0$ as

$$
\left|S_{I}^{\alpha}(f)\right|_{f=0}=\left\{\begin{array}{l}
\frac{A_{i}^{2}}{2 T_{i}}\left|Q\left(f_{i}+\frac{\alpha}{2}\right) Q^{*}\left(f_{i}-\frac{\alpha}{2}\right)\right|, \alpha=\frac{k}{T_{i}} \\
\frac{A_{i}^{2}}{4 T_{i}}\left|Q\left(\frac{\alpha}{2} \pm f_{i}\right)\right|^{2}, \alpha=\mp 2 f_{i}+\frac{k}{T_{i}} \\
0, \quad \text { otherwise. }
\end{array}\right.
$$

If we disregard $\alpha=0$ (in this case we cannot get any cyclic information from $\left.S_{I}^{\alpha}(f)\right)$, then from (11) we can find that $\left|S_{I}^{\alpha}(f)\right|_{f=0}$ takes the max values at $\alpha=2 f_{i}$, and this can be used to get the estimates of $f_{i}$, i.e.,

$$
\hat{f}_{i}=\frac{1}{2} \underset{\alpha(\alpha>0)}{\arg \max }\left(\left|S_{I}^{\alpha}(f)\right|_{f=0}\right) \text {. }
$$

Meanwhile, if we let $f=f_{i}$ in (10), we will have

$$
\left|S_{I}^{\alpha}(f)\right|_{f=f_{i}}=\left\{\begin{array}{l}
\frac{A_{i}^{2}}{4 T_{i}}\left|+\left(2 f_{i}+\frac{\alpha}{2}\right) Q^{*}\left(2 f_{i}-\frac{\alpha}{2}\right)\right|,\left.\alpha\left(\frac{\alpha}{2}\right)\right|^{2} \\
\frac{A_{i}^{2}}{4 T_{i}}\left|Q\left(2 f_{i}+\frac{\alpha}{2}\right) Q^{*}\left(\frac{\alpha}{2}\right)\right|, \alpha=\mp 2 f_{i}+\frac{k}{T_{i}} \\
0, \quad \text { otherwise. }
\end{array}\right.
$$

In (13), due that $Q(f)=\sin \left(\pi f T_{i}\right) /(\pi f)$, we can get that except the first right term takes the max value at $\alpha=1 / T_{i}$, all the other values are equal or approximate equal to zero for $\alpha \neq 1 / T_{i}$. Besides, from (11) we can also get that $\left|S_{I}^{\alpha}(f)\right|_{f=0}$ takes the submax values at $\alpha=$ $2 f_{i} \pm 1 / T_{i}$ (or max values if we discard the point $\alpha=2 f_{i}$ ). Combining these two results, we can get the estimate of data bit rate $R_{i}=1 / T_{i}$ as

$$
\hat{R}_{i}=\frac{1}{3}\left[\begin{array}{l}
\underset{\alpha, \alpha>0}{\arg \max }\left(\left|S_{I}^{\alpha}(f)\right|_{f=\hat{f}_{i}}\right)+\underset{\alpha, \alpha>2 \hat{f}_{i}}{\arg \max }\left(\left|S_{I}^{\alpha}(f)\right|_{f=0}\right) \\
+\underset{\alpha, 0<\alpha<2 \hat{f}_{i}}{\arg \max }\left(\left|S_{I}^{\alpha}(f)\right|_{f=0}\right)
\end{array}\right] .
$$

The results given in (12) and (14) indicate that by detecting the max value of SCF amplitude at $f=0$ (excluding point $\alpha=0$ ), we can easily get the estimate of carrier frequency $\hat{f}_{i}$, and by detecting the submax value of SCF amplitude at $f=0$ and max value at $f=\hat{f}_{i}$, we can get the estimate of data bit rate $\hat{R}_{i}$, thus we can get all the CFs of $i(t)$ [23], and they are critical to the following BAFRESH filter and RR-MSWF algorithm given in Sec. 3. Note that because $S_{x}^{-\alpha}(f)=S_{x}^{\alpha}(f)$, the estimated results of $\hat{f}_{i}$ and $\hat{R}_{i}$ by $\left|S_{x}^{-\alpha}(f)\right|$ are the same as the results by $\left|S_{x}^{\alpha}(f)\right|(\alpha>0)$, and we have ignored them.

\subsection{Obtaining of the Interference CFs from Cyclic Periodogram of the Received GPS Signal}

From Sec. 2.2 we have known that by CSA of the received GPS signal, we can get the estimates of the interference CFs. But in practice it is often difficult to achieve this because of the finite processed data. To this problem, we can use the time smoothing or frequency smoothing cyclic periodogram [15] instead of the SCF to get the estimates of the interference CFs. Since the time smoothing method is often considered more computationally efficient than the frequency smoothing method [18], we will choose the former to get the CFs of the interference. The discrete time smoothing cyclic periodogram of $x(n)$ can be given as [15]

$$
S_{X_{1 / \Delta f}}^{\alpha}(n, f)=\frac{1}{K P} \sum_{k=0}^{K P-1}\left\{\begin{array}{l}
\Delta f X_{1 / \Delta f}(n-k /(\Delta f K), f+\alpha / 2) \times \\
X_{1 / \Delta f}^{*}(n-k /(\Delta f K), f-\alpha / 2)
\end{array}\right\}
$$

where

$$
X_{1 / \Delta f}(k, f)=\sum_{i=0}^{N-1} a_{1 / \Delta f}(i) x(k-i) \exp \left[-\mathrm{j} 2 \pi f(k-i) T_{\mathrm{s}}\right],
$$

$P$ is the number of non-overlap data blocks, $K$ is a blockoverlap parameter, $a_{1 / \Delta f}(\bullet)$ is a data tapering window with the length of $N$ samples, $\Delta f=1 /\left[(N-1) T_{\mathrm{s}}\right]$ is the spectral resolution. In (15), the time length of total data segment $\Delta t=[(1+P-1 / K) N-1] T_{\mathrm{s}}$, and $0 \leq n \leq \Delta t / T_{\mathrm{s}}$.

To get the reliable estimates of the interference CFs, the product of $\Delta t$ and $\Delta f$ should meet the condition [15]

$$
\Delta t \Delta f=[(1+P-1 / K) N-1] /(N-1) \approx P \gg 1 .
$$

In practical applications, the implementation of (15) can be fulfilled by FFT accumulation method (FAM) or by strip spectral correlation analyzer (SSCA) [19]. Based on (12), (14) to (16), and using FAM algorithm, the process of obtaining the interference CFs of $\hat{f}_{i}$ and $\hat{R}_{i}$ can be given as following:

Step 1 Suppose $N$ is a power of 2 (if not, we can pad trailing zeros to the samples of $x(n)$ to realize this), based on (15) and (16), use FAM method to get $S_{X_{1 / \Delta f}^{\alpha}}^{\alpha}(n, f)$. 
Step 2 Get the position corresponding to the max value of $\left|S_{X_{1 / \alpha f}}^{\alpha}(n, f)\right|_{f=0}$, where $\alpha>0$. Suppose the result is $n_{f}$, we can get the estimated carrier frequency of interference as $\hat{f}_{i}=\left(n_{f}-n_{m}\right) /\left(N_{t} T_{S}\right)$, where $N_{t}=2 N^{2}$ is the total number of processed data by FAM in each data segment, and $n_{m}=N_{t} / 2$ is the middle position of $N_{t}$.

Step 3 For $\alpha>0$, find the positions corresponding to submax values of $\left|S_{X_{1 / \Delta f}}^{\alpha}(n, f)\right|_{f=0}$ in the ranges of $\left(n_{m}, n_{f}-3\right)$ and $\left(n_{f}+3, N_{t}\right)$, and suppose the results are $n_{b}^{(l)}$ and $n_{b}^{(r)}$, where $n_{b}^{(l)}$ and $n_{b}^{(r)}$ are on the left and right of $n_{f}$, respectively. Here we have empirically neglected 6 spectral lines which are centered at $n_{f}$ and may take the submax values due to cyclic spectral leakage [14].

Step 4 Find the positions corresponding to submax values of $\left|S_{X_{1 / \Delta f}}^{\alpha}(t, f)\right|_{f=\hat{f}_{i}}$ in the range $\left(n_{m}+3, N_{t}\right)$ and suppose the results is $n_{b}^{(f)}$, we can get the estimated data bit rate of interference as $\hat{R}_{i}=\left[\left(n_{b}^{(r)}-n_{m}\right)+\left(n_{m}-n_{b}^{(l)}\right)\right.$ $\left.+\left(n_{b}^{(f)}-n_{m}\right)\right] /\left(3 N_{t} T_{s}\right)$.

\section{Interference Mitigation with BA- FRESH Filtering and RR-MSWF Algorithm}

\subsection{Principles of BA-FRESH Filter}

With the interference CFs obtained from the cyclic periodogram of the received GPS signal, we can effectively mitigate the interference and separate the weak GPS signal with the adaptive FRESH filter. Due that the reference signal often cannot be got beforehand, we can use the blind adaptive FRESH (BA-FRESH) filter to fulfill the filtering. The structure of BA-FRESH filter [24] based on $x(n)$ of (1) is shown as Fig. 1, in which $\hat{I}(n)$ and $\tilde{s}_{\mathrm{g}}(n)$ are the estimated interference and the separated GPS signal, respectively.

In Fig. $1, \alpha_{\gamma} \in\left\{k \hat{R}_{i}\right\}(\gamma=1,2, \cdots, \Gamma)$ and $\beta_{\varsigma} \in$ $\left\{2 k \hat{f}_{i} \pm \hat{R}_{i}\right\}(\varsigma=1,2, \cdots, \Xi)$ are the non-conjugate and conjugate CFs of the interference, respectively, $k \in \mathbb{Z}$. For $v$ of the reference signal $d(n)$, it meets $v \in\left\{k \hat{R}_{i}\right\} U$ $\left\{2 k \hat{f}_{i} \pm \hat{R}_{i}\right\}(k \in \mathbb{Z})$ and $v \notin\left\{a_{\gamma}\right\}_{\gamma=1}^{\Gamma} \cup\left\{\beta_{\varsigma}\right\}_{\varsigma=1}^{\Xi}$. The results of $\hat{I}(n)$ and $\tilde{s}_{g}(n)$ can be given as [24], [25]

$$
\hat{I}(n)=\mathbf{h}^{\mathrm{H}} \tilde{\mathbf{x}}(n), \tilde{s}_{g}(n)=d(n)-\hat{I}(n)
$$

where the superscript $\mathrm{H}$ denotes transpose conjugate, and

$$
\begin{gathered}
\mathbf{h}=\left[\mathbf{h}^{\alpha_{1}}, \cdots, \mathbf{h}^{\alpha_{\Gamma}}, \mathbf{h}^{\beta_{1}}, \cdots, \mathbf{h}^{\beta_{\Xi}}\right]^{\mathrm{T}}, \\
\tilde{\mathbf{x}}(n)=\left[\mathbf{x}^{\alpha_{1}}(n), \mathbf{x}^{\alpha_{2}}(n), \cdots, \mathbf{x}^{\alpha_{\Gamma}}(n), \mathbf{x}^{\beta_{1}}(n), \mathbf{x}^{\beta_{2}}(n), \cdots \mathbf{x}^{\beta_{\Xi}}(n)\right]^{\mathrm{T}}
\end{gathered}
$$

where the superscript $\mathrm{T}$ denotes transpose, $\mathbf{h}^{\alpha_{y}}$ and $\mathbf{x}^{\alpha_{y}}(n)$ (or $\mathbf{h}^{\beta_{\varsigma}}$ and $\mathbf{x}^{\beta_{\varsigma}}(n)$ ) are respectively the coefficients and inputs of the FIR filter corresponding to $\alpha_{\gamma}$ with $L_{\gamma}$ taps (or $\beta_{\varsigma}$ with $L_{\varsigma}$ taps),

$\left\{\begin{array}{l}\mathbf{h}^{\alpha_{\gamma}}=\left[h^{\alpha_{\gamma}}(0), h^{\alpha_{\gamma}}(1), \cdots, h^{\alpha_{\gamma}}\left(L_{\gamma}-1\right)\right]^{\mathrm{T}}, \quad \gamma=1,2, \cdots \Gamma \\ \mathbf{h}^{\beta_{\zeta}}=\left[h^{\beta_{\varsigma}}(0), h^{\beta_{\varsigma}}(1), \cdots, h^{\beta_{\varsigma}}\left(L_{\varsigma}-1\right)\right]^{\mathrm{T}}, \quad \varsigma=1,2, \cdots \Xi\end{array}\right.$

and

$$
\left\{\begin{aligned}
\mathbf{x}^{\alpha_{\gamma}}(n)= & {\left[x\left(n-L_{\gamma}+1\right) \exp \left(\mathrm{j} 2 \pi \alpha_{\gamma}\left(n-L_{\gamma}+1\right)\right), \cdots,\right.} \\
& \left.x(n) \exp \left(\mathrm{j} 2 \pi \alpha_{\gamma} n\right)\right]^{\mathrm{T}}, \\
\gamma & =1,2, \cdots \Gamma, \\
\mathbf{x}^{\beta_{\zeta}}(n)= & {\left[x^{*}\left(n-L_{\varsigma}+1\right) \exp \left(\mathrm{j} 2 \pi \beta_{\varsigma}\left(n-L_{\varsigma}+1\right)\right), \cdots,\right.} \\
& \left.x^{*}(n) \exp \left(\mathrm{j} 2 \pi \beta_{\varsigma} n\right)\right]^{\mathrm{T}}, \\
\varsigma & 1,2, \cdots \Xi .
\end{aligned}\right.
$$

\subsection{Implementation of BA-FRESH Filtering with RR-MSWF Algorithm}

In practice, we can implement the BA-FRESH filtering with recursive least squares (RLS) algorithm, least mean square (LMS) algorithm and so on [24]. Considering the computation efficiency, in the following numerical simulations we have used the efficient RR-MSWF algorithm [21], [22] to mitigate the interference and separate the GPS signal. The schematic diagram of RR-MSWF algorithm based on Fig. 1 is shown as Fig. 2.

Based on Fig. 2 and equations (18) to (22), the detailed implementation of the interference mitigation with the batch adaptive RR-MSWF algorithm [21] is given as in Tab. 1 .

\section{Numerical Results}

To show the effectiveness of the proposed method, simulations shown as Figs. 3 to 8 are offered for verification. Among them, Figure 3 offers CSA results of the received GPS C/A code signal overlapped with the strong interference, Figures 4 to 8 offer the acquisition, probability of detection, and tracking of the separated weak GPS signal with the proposed method, respectively, in which the CFs $\hat{f}_{i}$ and $\hat{R}_{i}$ of the BA-FRESH filter used in Figs. 4 to 6 are obtained from Figs. 3 and the CFs used in Figs. 7 to 8 are got in a similar way given as Fig. 3. Besides, in Figs. 4 to 8 simulations with the conventional MF filtering are offered for comparison, in Figs. 5 and 6 simulation results without the strong interference are offered for further comparison, and in Figs. 7 and 8 the cases in the presence of more strong interference are also offered for further verification. 


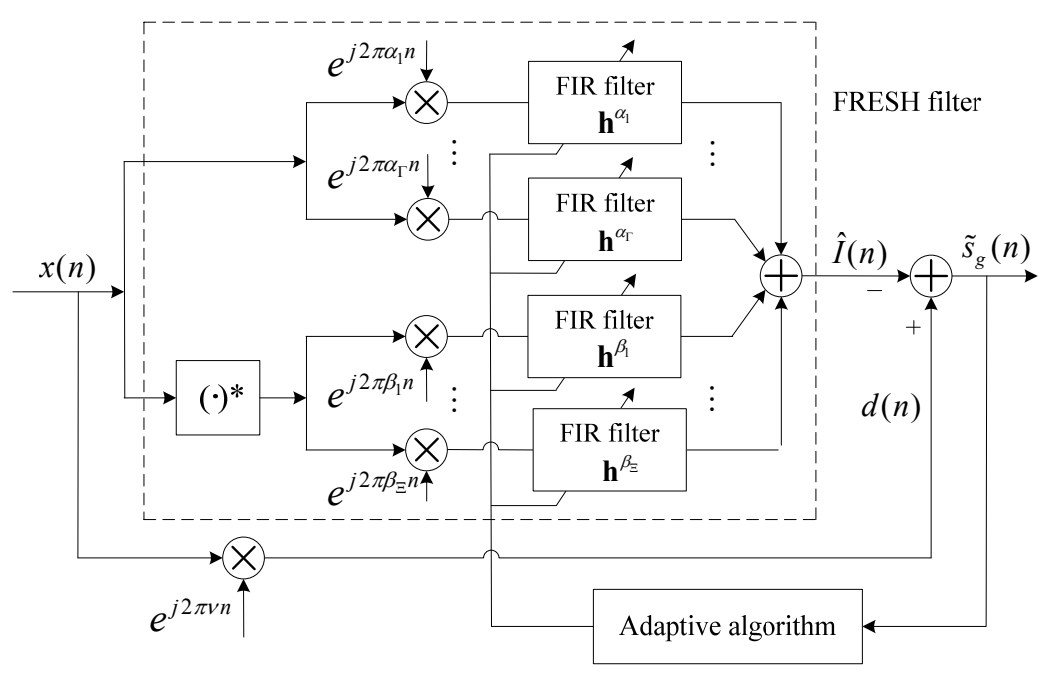

Fig. 1. Schematic diagram of BA-FRESH filter.



Fig. 2. Schematic diagram of RR-MSWF algorithm (stage $D=3$ ).

\begin{tabular}{|c|c|}
\hline \multicolumn{2}{|c|}{$\begin{array}{l}\text { Initialization: } \\
\mathbf{X}_{0}=\left[\tilde{\mathbf{x}}(1), \tilde{\mathbf{x}}(2), \cdots, \tilde{\mathbf{x}}(k), \cdots, \tilde{\mathbf{x}}\left(N_{\Delta t}\right)\right], \\
\mathbf{d}_{0}=\left[d(1), d(2), \cdots, d(k), \cdots, d\left(N_{\Delta t}\right)\right] .\end{array}$} \\
\hline $\begin{array}{l}\text { Forward recursion: } \\
\qquad \begin{aligned} \text { for } r=1,2, \cdots, D \\
\boldsymbol{\lambda}_{r}=\mathbf{X}_{r-1} \mathbf{d}_{r-1}^{H} ; \\
\mathbf{h}_{r}=\boldsymbol{\lambda}_{r} /\left\|\boldsymbol{\lambda}_{r}\right\| ; \\
\mathbf{d}_{r}=\mathbf{h}_{r}^{H} \mathbf{X}_{r-1} ; \\
\mathbf{B}_{r}=\mathbf{I}-\mathbf{h}_{r} \mathbf{h}_{r}^{H} ; \\
\mathbf{X}_{r}=\mathbf{B}_{r}^{H} \mathbf{X}_{r-1} ;\end{aligned} \\
\text { end }\end{array}$ & $\begin{array}{l}\text { Backward recursion: } \\
\qquad \begin{array}{l}\boldsymbol{\varepsilon}_{D}=\mathbf{d}_{D} ; \\
\text { for } r=D, D-1, \cdots, 1 \\
\qquad w_{r}=\boldsymbol{\varepsilon}_{r} \mathbf{d}_{r-1}^{H} ; \\
\boldsymbol{\varepsilon}_{r-1}=\mathbf{d}_{r-1}-w_{r}^{*} \boldsymbol{\varepsilon}_{r} ;\end{array} \\
\text { end }\end{array}$ \\
\hline
\end{tabular}

Output of the separated GPS signal:

$\tilde{\mathbf{s}}_{g}=\boldsymbol{\varepsilon}_{0}=\mathbf{d}_{0}-w_{1}^{*} \boldsymbol{\varepsilon}_{1}$

where

$\tilde{\mathbf{s}}_{g}=\left[\tilde{s}_{g}(1), \tilde{s}_{g}(2), \cdots, \tilde{s}_{g}(k), \cdots, \tilde{s}_{g}\left(N_{\Delta t}\right)\right]$.

Tab. 1. Implementation of BA-FRESH filtering with the batch adaptive RR-MSWF algorithm to mitigate the strong interference. In the table, $N_{\Delta t}$ is the total samples input the BA-FRESH filter in time interval $\Delta t, \mathbf{I}$ is a unit matrix, and $\|\cdot\|$ denotes 2-norm.

The common parameter setups used in Figs. 3 to 8 are given as following:

- Time length $\Delta t=1 \mathrm{~ms}$ except in Figs. 6 and 8 it is set to $40 \mathrm{~ms}$, and sampling frequency $f_{\mathrm{s}}=1 / T_{\mathrm{s}}=16 \mathrm{MHz}$.

- Carrier IF, Doppler shift and initial C/A code phase of the GPS signal are respectively set as $f_{\mathrm{IF}}=$
4.309 MHz, $f_{\mathrm{d}}=2 \mathrm{kHz}$, and $\tau_{\mathrm{g}}=200$ chips or 3128 in samples.

- In Figs. 4 to 6 , frequency bias and data bit rate of interference are respectively set as $\Delta f_{i}=500 \mathrm{kHz}$ and $R_{i}=1.544 \mathrm{Mbit} / \mathrm{s}$; while in Figs. 7 and 8, the setups of different type of interference are given in Tab. 2. 
Other different parameter setups used in each simulation are given as following:

- In Fig. 4, while using the FAM algorithm to get the CFs of the interference, the cycle resolution $\Delta \alpha=1 / \Delta t$ is set to $1 \mathrm{kHz}$ and the spectral resolution $\Delta f$ is set to $120 \mathrm{kHz}, K$ is chosen to be 4 according to [18];

- In Figs. 4 to 8, for the BA-FRESH filtering, $\alpha_{\gamma} \in$ $\left\{ \pm \hat{R}_{i}\right\}, \beta_{\varsigma} \in\left\{ \pm 2 \hat{f}_{i}, \pm 2 \hat{f}_{i} \pm \hat{R}_{i}\right\}$, and $v=0$ for the reference signal [24], $L_{\gamma}=L_{\varsigma}=10(\gamma=1,2 ; \varsigma=1$, $2, \cdots, 6)$, and $D$ of RR-MSWF algorithm is chosen to be 8 according to the result given in [21].

- In Figs. 5 and 7, the probability of detection is calculated by Monte Carlo method and the independent run number is 300 , and $P_{\mathrm{fa}}$ in the figure is the false-alarm probability;

- In Figs. 6 and 8, the root mean square error (RMSE) of each tracking result is the average of 50 independent runs.

\subsection{CSA of the Received GPS Signal}

From Fig. 3(a) we can get that the estimated carrier frequency, and two estimated data bit rates respectively as $\hat{f}_{i}=9.6182 / 2=4.8091 \mathrm{MHz}, R_{i}^{(l)}=9.6182-8.0742=$ $1.544 \mathrm{Mbit} / \mathrm{s}$ and $R_{i}^{(r)}=11.1622-9.6182=1.544 \mathrm{Mbit} / \mathrm{s}$. From Fig. 3(b) we can get $R_{i}^{(0)}=1.544 \mathrm{Mbit} / \mathrm{s}$. Then from these results we can get the final estimated data bit rate as $\hat{R}_{i}=\left(R_{i}^{(l)}+R_{i}^{(r)}+R_{i}^{(0)}\right) / 3=1.544 \mathrm{Mbit} / \mathrm{s}$. All these results show that they fit well with their preset values.

\subsection{Acquisition and Detection of the Separated GPS Signal}

From Fig. 4(a) we can see that when the strength of interference equals $5 \mathrm{~dB}$, i.e., the interference is relatively weak, we may still acquire the weak GPS signal with the conventional MF filtering; but when the strength of interference increases to $15 \mathrm{~dB}$ as given in Fig. 4(b), i.e., the interference becomes relatively stronger, we almost cannot acquire the weak GPS signal. But on the other hand, in these two cases, we can well acquire both the weak GPS signals with the proposed method (denoted by "BAF-RR" filtering).

From Fig. 5 we can further learn the difference of two methods for the acquisition of the weak GPS signal from their probabilities of detection. From Fig. 5(a), we can see that when the strength of interference equals $5 \mathrm{~dB}$, and under the condition that probability of detection $P_{\mathrm{d}}=0.8$, the proposed BAF-RR filtering outperforms the MF filtering about $4 \mathrm{~dB}$ for $P_{\mathrm{fa}}=10^{-3}$, and $6 \mathrm{~dB}$ for $P_{\mathrm{fa}}=10^{-6}$, and is only about $2 \mathrm{~dB}$ lower than the result without the strong interference for $P_{\mathrm{fa}}=10^{-3}$ or $P_{\mathrm{fa}}=10^{-6}$. While from Fig. 5(b) we also can see that when the strength of interference equals $12 \mathrm{~dB}$, under the same condition that $P_{\mathrm{d}}=0.8$, the proposed BAF-RR filtering outperforms the MF filter-

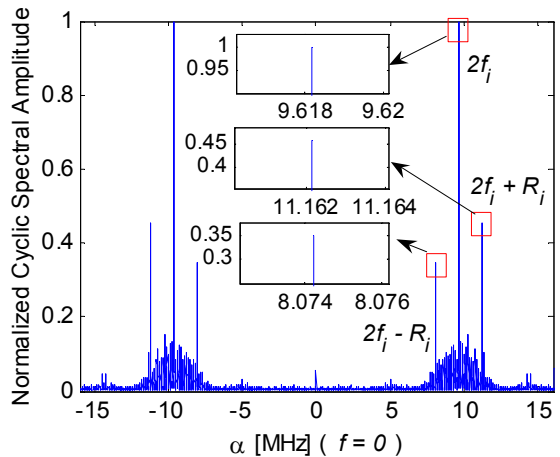

(a)

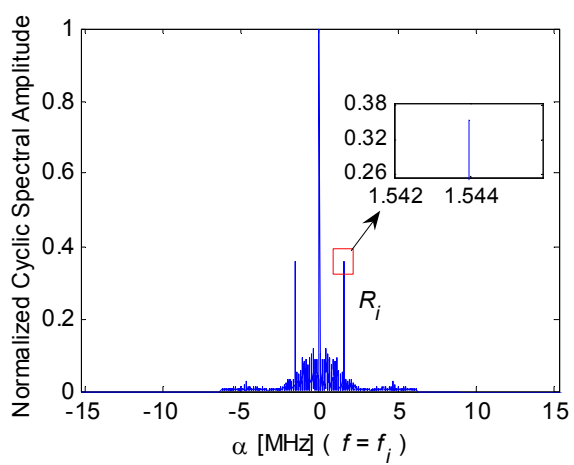

(b)

Fig. 3. Obtain the estimates of the strong interference CFs $R_{i}$ and $f_{i}$ from the special planes of cyclic periodogram, in which (a) is the plane at $f=0$, and (b) is the plane at $f=f_{i}$. The signal to noise ratios (SNRs) of the GPS and the interference are $-15 \mathrm{~dB}$ and $10 \mathrm{~dB}$, respectively.
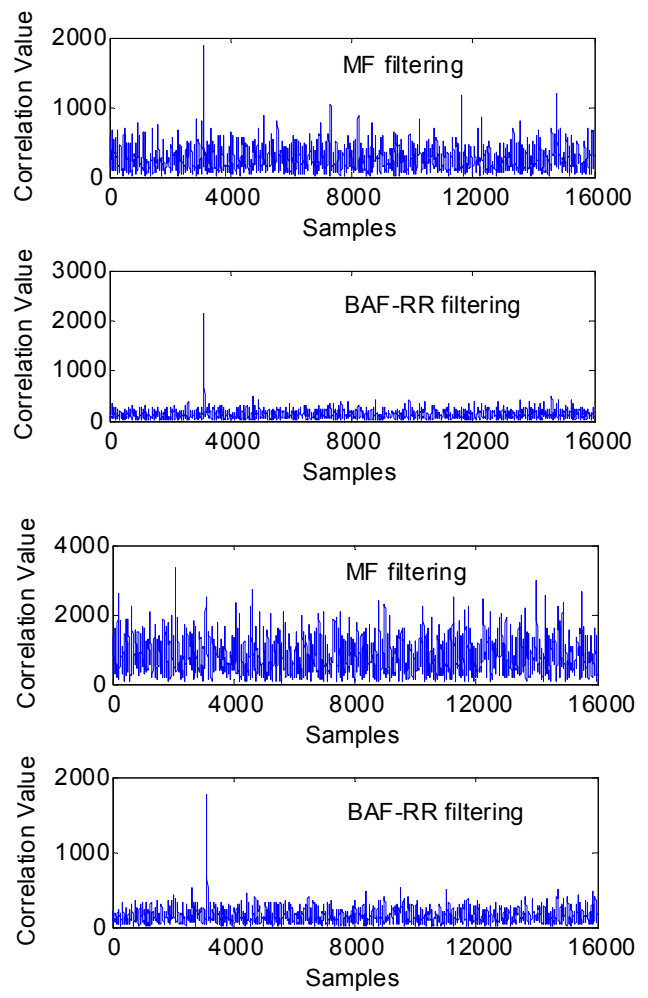

Fig. 4. Acquisition comparisons of the separated GPS signal filtered with the conventional MF method and with the proposed BAF-RR method, in which the SNR of the GPS signal is $-15 \mathrm{~dB}$ and the SNRs of the strong interference are (a) $5 \mathrm{~dB}$ and (b) $15 \mathrm{~dB}$, respectively. 


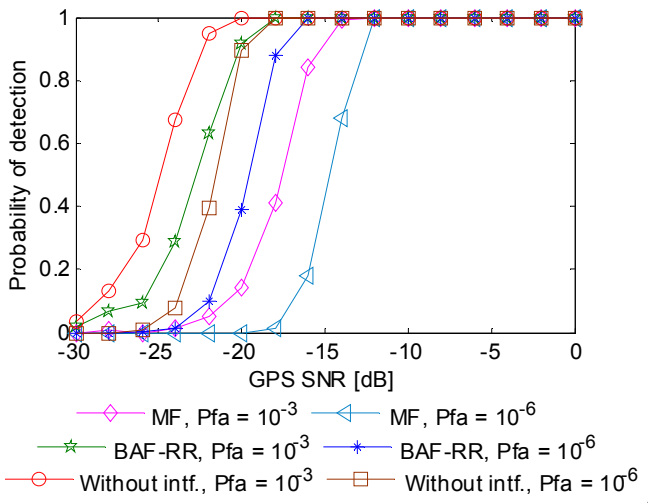

(a)

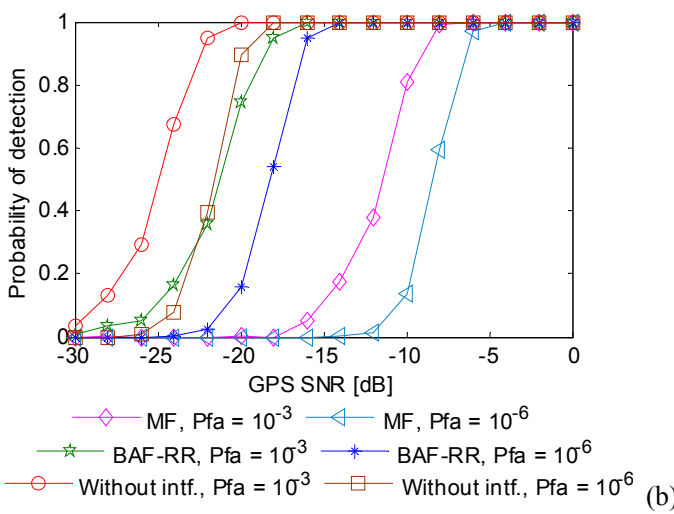

Fig. 5. Comparisons of detection probability of the separated GPS signal filtered with the conventional MF method and with the proposed BAF-RR method, in which the SNRs of the strong interference are (a) $5 \mathrm{~dB}$ and (b) $12 \mathrm{~dB}$, respectively.

ing about $7 \mathrm{~dB}$ for $P_{\mathrm{fa}}=10^{-3}$ and $10 \mathrm{~dB}$ for $P_{\mathrm{fa}}=10^{-6}$, and is only about $3 \mathrm{~dB}$ lower than the result without the strong interference for $P_{\mathrm{fa}}=10^{-3}$, and $4 \mathrm{~dB}$ for $P_{\mathrm{fa}}=10^{-6}$. In addition, under the condition that $P_{\mathrm{d}}=0.8$, with the proposed BAF-RR filtering we can detect the weak GPS signal as low as $-24 \mathrm{~dB}$ for the SNR of overlapped interference equal to $5 \mathrm{~dB}$, and $-20 \mathrm{~dB}$ for the SNR of interference equal to $12 \mathrm{~dB}$. The results given in Fig. 5 are also consistent with the results given in Fig. 4.

\subsection{Tracking of the Separated GPS Signal}

The tracking of the GPS signal is the another critical stage of the GPS receiver, and it is mainly fulfilled by the discriminator of delay locked loop (DLL) aided with the carrier phase locked loop (PLL). Here we use the discriminator whose tracking error is given by $\Delta \tau=\left(\sqrt{I_{\mathrm{ES}}^{2}+Q_{\mathrm{ES}}^{2}}-\sqrt{I_{\mathrm{LS}}^{2}+Q_{\mathrm{LS}}^{2}}\right) /\left(\sqrt{I_{\mathrm{ES}}^{2}+Q_{\mathrm{ES}}^{2}}+\sqrt{I_{\mathrm{LS}}^{2}+Q_{\mathrm{LS}}^{2}}\right)$

[28] to measure the DLL tracking performance of the separated GPS signal, where $I_{\mathrm{ES}}$ and $I_{\mathrm{LS}}$ are respectively the coherent integrations of early (E) and late (L) correlators of the in-phase, $Q_{\mathrm{ES}}$ and $Q_{\mathrm{LS}}$ are the similar results of the quadrature-phase. The parameter dEL in Fig. 6 is the spacing of $\mathrm{E}$ and L correlators.

From Fig. 6 we can see that with the BAF-RR filtering, we can also improve the tracking performance of the

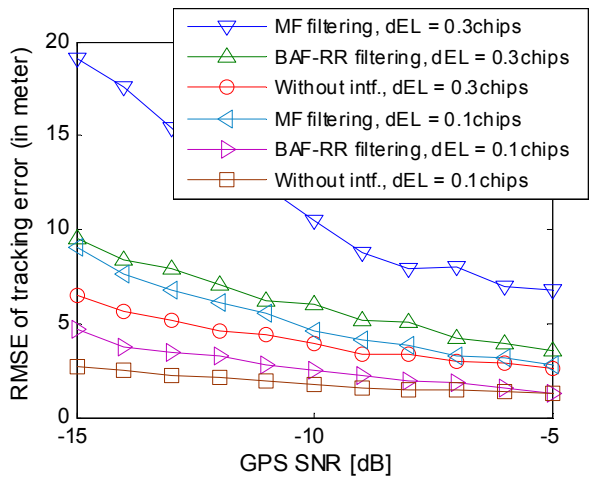

(a)

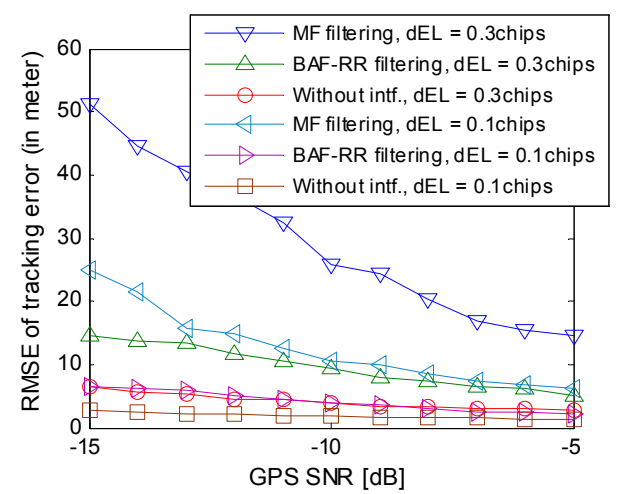

(b)

Fig. 6. RMSE comparisons of the tracking of the separated GPS signal filtered with the conventional MF method and with the proposed BAF-RR method, in which the SNRs of the strong interference are (a) $5 \mathrm{~dB}$ and (b) $12 \mathrm{~dB}$, respectively.

separated GPS signal dramatically. For example, in Fig. 6(a) when the strength of the interference equals $5 \mathrm{~dB}$, under the condition that GPS SNR $=-13 \mathrm{~dB}$ and $\mathrm{dEL}=0.3$ chips, for the conventional MF filtering, RMSE of tracking error of the separated GPS signal is about $18 \mathrm{~m}$; but after the interference mitigation with the proposed BAF-RR filtering, the RMSE of tracking error of the separated GPS signal will decrease to about $9 \mathrm{~m}$. In Fig. 6(b) we can also get the tracking performance of the separated GPS signal filtered with the proposed BAF-RR filtering greatly outperforms that with the conventional MF filtering, and the tracking result with the proposed BAF-RR method is nearly close to the case without the strong interference.

In addition, from Fig. 6 we can also see that the dEL also has an important effect on the tracking accuracy of the GPS receiver. Generally speaking, the correlators of E and $\mathrm{L}$ with small dEL are preferable to those with large dEL in improving the tracking accuracy of the GPS receiver.

\subsection{Acquisition and Tracking Performance of the Separated GPS Signal in the Presence of More Strong Interference}

In this section, the acquisition and tracking performance of the separated GPS signal in the presence of more strong interference is also verified. The simulation results are shown as Figs. 7 and 8 respectively, in which the setups of different interference are given in Tab. 2. 


\begin{tabular}{|c|c|c|c|}
\hline Interference type & $\begin{array}{c}\text { SNR } \\
(\mathrm{dB})\end{array}$ & $\begin{array}{c}\text { Carrier frequency } \\
(\mathrm{MHz})\end{array}$ & Bit or chirp rate \\
\hline BPSK & 10 & $f_{i}=f_{\mathrm{IF}}+\Delta f_{i}=4.809$ & $R_{i}=1.544 \mathrm{Mbps}$ \\
\hline $\begin{array}{c}\text { Binary amplitude } \\
\text { shift keying } \\
\text { (2ASK) }\end{array}$ & 7 & $f_{i}=4.609$ & $R_{i}=1.2288 \mathrm{Mbps}$ \\
\hline $\begin{array}{c}\text { Binary frequency } \\
\text { shift keying (2FSK) }\end{array}$ & 5 & $\begin{array}{c}f_{i 1}=4.109, \\
f_{i 2}=4.189\end{array}$ & $R_{i}=1.2288 \mathrm{Mbps}$ \\
\hline $\begin{array}{c}\text { Linear frequency } \\
\text { modulation (LFM } \\
\text { or chirp) }\end{array}$ & 5 & $\begin{array}{c}f_{i}=3.909 \\
\text { (initial frequency) }\end{array}$ & $\begin{array}{c}k=6.7 \times 10^{7} \mathrm{~Hz} / \mathrm{s} \\
B=0.2 \mathrm{MHz}\end{array}$ \\
\hline
\end{tabular}

Tab. 2. The setups of different interference in which $f_{\mathrm{IF}}=4.309 \mathrm{MHz}, k$ and $B$ of LFM are frequency change rate and bandwidth respectively.
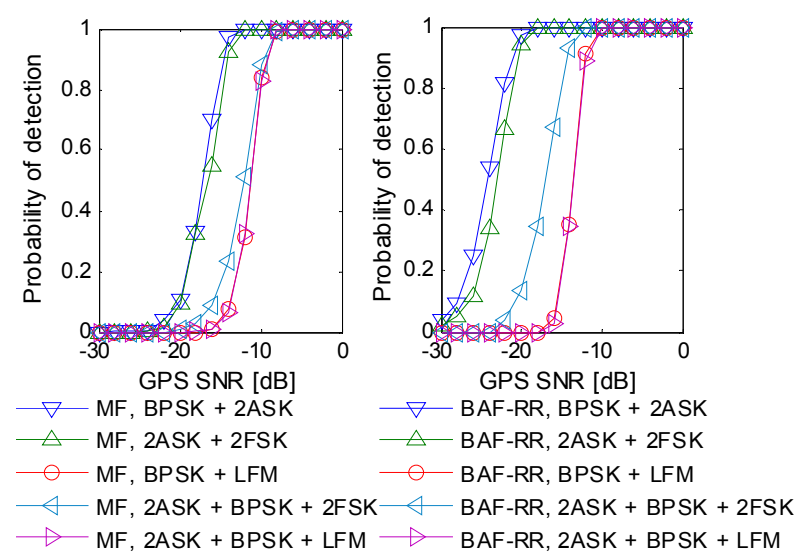

Fig. 7. Comparisons of detection probability of the separated GPS signal filtered with the conventional MF method and with the proposed BAF-RR method under more strong interference, in which $P_{\mathrm{fa}}=10^{-3}$ and the setups of the interference are given in Tab. 2.
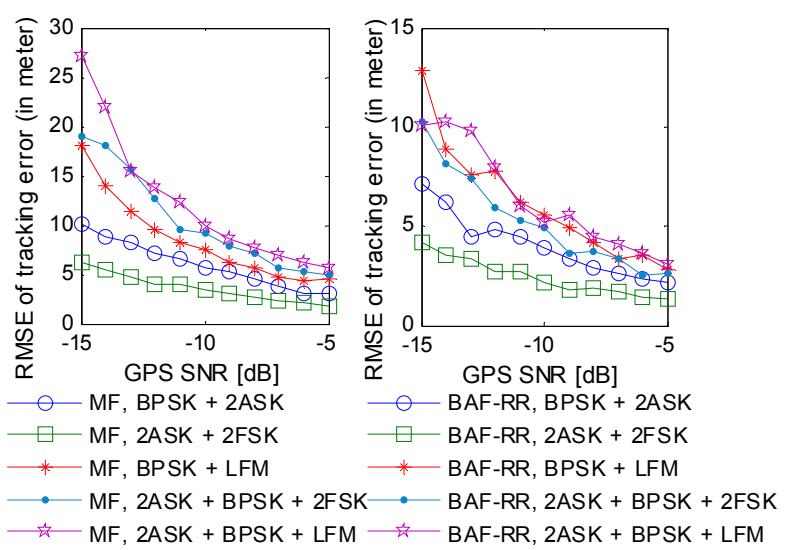

Fig. 8. RMSE comparisons of the tracking of the separated GPS signal filtered with the conventional MF method and with the proposed BAF-RR method under more strong interference, in which $\mathrm{dEL}=0.1$ chips and the setups of the interference are given in Tab. 2.

From Fig. 7 we can get that under more strong interference, for the same $P_{\mathrm{d}}$ of the separated GPS signal, the proposed BAF-RR algorithm still outperforms the MF algorithm greatly in GSP SNR. For example, for $P_{\mathrm{d}}=0.8$ and the case of two interference of BPSK + 2ASK, BAR$\mathrm{RR}$ can outperform MF about $6 \mathrm{~dB}$ in GPS SNR. On the other hand, from Fig. 7 we also can get that for the two algorithms, when there exists the LFM interference, their acquisition performance will all degrade dramatically, and this can be explained by that the GPS signal and the given LFM interference almost cannot be distinguished in both correlation domain and cyclic spectral domain. But even for this worst case, the proposed BAR-RR algorithm still can filter some more LFM interference than the conventional MF algorithm, as can be seen from their comparisons of $P_{\mathrm{d}}$ in Fig. 7.

While from Fig. 8 we can see that when there is more strong interference, with the proposed BAR-RR algorithm we can also improve the tracking accuracy of the separated GPS signal a lot than with the conventional MF algorithm. For example, when GPS SNR $=-15 \mathrm{~dB}$ and for the case of three interference of $2 \mathrm{ASK}+\mathrm{BPSK}+2 \mathrm{FSK}$, RMSE of tracking error of the separated GPS signal is about $10 \mathrm{~m}$, while for the conventional MF algorithm the result is about $20 \mathrm{~m}$. In addition, from Fig. 8 we can also get that when there exists the LFM interference, the tracking performance of both MF algorithm and BAR-RR algorithm will degrade greatly, and this is just well consistent with the result given in Fig. 7.

\section{Conclusions}

A method based on CSA and RR-MSWF algorithm to mitigate the strong interference for the GPS receiver is proposed. Utilizing the CFs obtained by CSA of the received GPS signal and BA-FRESH filtering combined with the efficient RR-MSWF algorithm, we can effectively mitigate the strong interference overlapping on the weak GPS signal in both time and frequency domains, and hence improve the acquisition and tracking performance of the GPS receiver dramatically. On the other hand, due that the delayed signals input the FRESH filter are complex variables, the computation of BA-FRESH filtering is often more complicated than that of the conventional filtering. Concretely, based on (18)-(22) and RR-MSWF algorithm offered in Tab. 1, the computation complexity of BAFRESH filtering can be given as about $O\left(N_{\Delta t}\left(\Gamma L_{\gamma}+\Xi L_{\varsigma}\right) D\right)$, while for the conventional FIR or MF filtering, the result is about $O\left(N_{\Delta t} \ell\right)$, where $\ell$ is the number of FIR filter taps or the Doppler shift searching step size of MF filtering, and often $\ell<\left(\Gamma L_{\gamma}+\Xi L_{\varsigma}\right) D$, correspondingly the computation time of the proposed method will be larger than that of the conventional one. For example, with the values of $\Gamma=6$, $\Xi=2, L_{\gamma}, L_{\varsigma}, D$ and $f_{\mathrm{s}}$ given in the beginning of Sec. 4 , suppose CPU operation frequency is $1.0 \mathrm{GHz}$ and $\Delta t=$ $1 \mathrm{~ms}$ and $\ell=50$, the time cost of MF is about $1.6 \mathrm{~ms}$ and the proposed method is about $10.2 \mathrm{~ms}$. Often, to this problem of complexity or computation time, we can make a tradeoff between the number of CFs $(=\Gamma+\Xi)$ and taps $L_{\gamma}$ (or $L_{\varsigma}$ ) used in the FRESH filter and the performance of the separated GPS signal. 


\section{Acknowledgments}

This work was supported by the Initial Research Fund of Chuzhou University under grant 2015qd05 and NSFC under grant 61571224. The authors would also like to acknowledge the anonymous reviewers for their helpful comments.

\section{References}

[1] WILDEMEERSCH, M., FORTUNY GUASCH, J. Radio frequency interference impact assessment on global navigation satellite systems. European Commission Joint Research Center, Science and Technique Report, JRC55767, 2010. DOI: $10.2788 / 6033$

[2] JAHROMI, A. J., BROUMANDAN, A., DANESHMAND, S., et al. Vulnerability analysis of civilian L1/E1 GNSS signals against different types of interference. In Proceedings of the 28th International Technical Meeting of the ION Satellite Division. Tampa, Florida (USA), 2015, p. 3262-3271.

[3] LI, J., NIE, J., LI, B., et al. Increase of carrier-to-noise ratio in GPS receivers caused by continuous-wave interference. Radioengineering, 2016, vol. 25, no. 3, p. 506-517. DOI: 10. 13164/re.2016.0506

[4] SAVASTA, S., PRESTI, L. L., RAO, M. Interference mitigation in GNSS receivers by a time-frequency approach. IEEE Transactions on Aerospace and Electronic Systems, 2013, vol. 49, no. 1, p. 415 to 438. DOI: $10.1109 /$ taes.2013.6404112

[5] ANYAEGBU, E., BORDIN, G., COOPER, J., et al. An integrated pulsed interference mitigation for GNSS receivers. The Journal of Navigation, 2008, vol. 61, no. 2, p. 239-255. DOI: $10.1017 / \mathrm{s} 0373463307004572$

[6] RAGHAVAN, S., LAZAR, S., EDGAR, C., et al. Cochannel and adjacent channel interference to GPS use. In 17th AIAA International Communications Satellite Systems Conference and Exhibit. 1998, p. 564-574. DOI: 10.2514/6.1998-1330

[7] LI, M., DEMPSTER, A. G., BALAEI, A. T., et al. Switchable beam steering/null steering algorithm for $\mathrm{CW}$ interference mitigation in GPS C/A code receivers. IEEE Transactions on Aerospace and Electronics Systems, 2011, vol. 47, no. 3, p. 1564-1579. DOI: $10.1109 /$ taes.2011.5937250

[8] MAO, W. L. GPS interference mitigation using derivative-free Kalman filter-based RNN. Radioengineering, 2016, vol. 25, no. 3, p. 518-526. DOI: 10. 13164/re.2016.0518

[9] HOU, Y. G., GUO, W., LI, X. S. Design of a GPS receiver for the linear frequency modulation interference suppression. In IEEE International Conference on Communications, Circuits and Systems. 2009, p. 454-456. DOI: 10.1109/icccas.2009.5250504

[10] BHUIYAN, M. Z. H., KUUSNIEMI, H., SÖDERHOLM, S., et al. The impact of interference on GNSS receiver observables - a running digital sum based simple jammer detector. Radioengineering, 2014, vol. 23, no. 3, p. 898-906.

[11] LEE, Y. T., CHANG, C. M., MAO, W. L., et al. Matched-filterbased low-complexity correlator for simultaneously acquiring global positioning system satellites. IET Radar, Sonar and Navigation, 2010, vol. 4, no. 5, p. 712-723. DOI: 10.1049/ietrsn.2009.0147

[12] FANTE, R., VACCARO, J. J. Wideband cancellation of interference in a GPS receiver array. IEEE Transactions on Aerospace and Electronic Systems, 2000, vol. 36, no. 2, p. 549-564. DOI: $10.1109 / 7.845241$

[13] CAPPOZZA, P. T., HOLLAND, B. J., HOPKINSON, T. M., et al A single-chip narrow-band frequency-domain excisor for a global positioning system (GPS) receiver. IEEE Journal of Solid-State Circuits, 2000, vol. 35, no. 3, p. 401-411. DOI: 10.1109/4.826823

[14] MUSUMECI, L., DOVIS, F. Performance assessment of wavelet based techniques in mitigating narrow-band interference In IEEE International Conference on Localization and GNSS (ICL-GNSS). 2013, p. 1-6. DOI: 10.1109/icl-gnss.2013.6577264

[15] GARDNER, W. A. Measurement of spectral correlation. IEEE Transactions on Acoustics, Speech and Signal Processing, 1986, vol. 34, no. 5, p. 1111-1123. DOI: 10.1109/tassp.1986.1164951

[16] ŠEBESTA, V., MARŠÁLEK, R., FEDRA, Z. OFDM signal detector based on cyclic autocorrelation function and its properties. Radioengineering, 2011, vol. 20, no. 4, p. 926-931.

[17] DIMC, F., BALDINI, G., KANDEEPAN, S. Experimental detection of mobile satellite transmissions with cyclostationary features. International Journal of Satellite Communications and Networking, 2015, vol. 33, no. 2, p. 163-183. DOI: 10.1002 /sat.1081

[18] ROBERTS, R. S., BROWN, W. A., LOOMIS, H. H. Computationally efficient algorithms for cyclic spectral analysis. IEEE Signal Processing Magazine, 1991, vol. 8, no. 2, p. 38-49. DOI: $10.1109 / 79.81008$

[19] NAPOLITANO, A., PERNA, I. Cyclic spectral analysis of the GPS signal. Digital Signal Processing, 2014, vol. 33, p. 13-33. DOI: $10.1016 /$ j.dsp.2014.06.003

[20] HUANG, P., PI, Y., PROGRI, I. GPS signal detection under multiplicative and additive noise. The Journal of Navigation, 2013, vol. 66, no. 4, p. 479-500. DOI: 10.1017/s0373463312000550

[21] HONIG, M. L., GOLDSTEIN, J. S. Adaptive reduced-rank interference suppression based on the multistage Wiener filter. IEEE Transactions on Communications, 2002, vol. 50, no. 6, p. 986-994. DOI: $10.1109 /$ tcomm.2002.1010618

[22] SONG, N., DE LAMARE, R. C., HAARDT, M., et al. Adaptive widely linear reduced-rank interference suppression based on the multistage Wiener filter. IEEE Transactions on Signal Processing, 2012, vol. 60, no. 8, p. 4003-4016. DOI: $10.1109 /$ tsp. 2012.2197747

[23] GARDNER, W. A. Cyclic Wiener filtering: theory and method. IEEE Transactions on Communications, 1993, vol. 41, no. 1 , p. 151-163. DOI: $10.1109 / 26.212375$

[24] ZHANG, J., WONG, K. M., LUO, Z. Q., et al. Blind adaptive FRESH filtering for signal extraction. IEEE Transactions on Signal Processing, 1999, vol. 47, no. 5, p. 1397-1402. DOI: $10.1109 / 78.757230$

[25] HU, Y., SONG, M., MENG, B. GPS signal availability augmentation utilizing the navigation signal retransmission via the GEO comsat. Wireless Personal Communications, 2015, vol. 82, no. 4, p. 2655-2671. DOI: $10.1007 / \mathrm{s} 11277-015-2371-9$

[26] DOBRE, O. A., ABDI, A., BAR-NESS, Y., et al. Cyclostationarity based blind classification of analog and digital modulations. In Proceedings of the 2006 IEEE Conference on Military Communications MILCOM 2006. Washington (USA), 2006, p. 2176-2182. DOI: $10.1109 /$ milcom.2006.302556

[27] RAMKUMAR, B. Automatic modulation classification for cognitive radios using cyclic feature detection. IEEE Circuits and Systems Magazine, 2009, vol. 9, no. 2, p. 27-45. DOI: $10.1109 /$ mcas.2008.931739

[28] KAPLAN, E. D., HEGARTY, C. J. Understanding GPS: Principles and Applications. $2^{\text {nd }}$ ed., Norwood (USA): Artech House, 2006. ISBN: 1580538940 


\section{About the Authors ...}

Yi HU was born in Lu'an, China, in 1974. He is currently a lecturer and engineer in communication engineering in Chuzhou University. He received his Ph.D. degree from Nanjing University of Aeronautics \& Astronautics (NUAA) in 2015. His research interests include satellite navigation signal processing and Sat-COM.

Maozhong SONG was born in Shexian, China, in 1962. $\mathrm{He}$ is a professor in the College of Electronic \& Information Engineering, NUAA. His current research interests include satellite navigation signal processing, communication measurement and controlling techniques.

Xiaoyu DANG was born in Nanjing, China, in 1973. He is a professor in the College of Electronic \& Information Engineering, NUAA. His current research interests include communication signal processing and Sat-COM.

Hongli YAN was born in Chuzhou, China, in 1981. She is a lecturer in Chuzhou University. She received her M.Sc. degree from Wuhan University of Science and Technology in 2008. Her current research interests include wireless communications and signal processing. 